\title{
Evaluation of the correlation between KRAS mutated allele frequency and pathologist tumorous nuclei percentage assessment in colorectal cancer suggests a role for zygosity status
}

\author{
Louis Libbrecht, ${ }_{1}^{1}$ Pamela Baldin, ${ }^{1}$ Anne-France Dekairelle, ${ }^{2}$ Anne Jouret-Mourin ${ }^{1}$
}

${ }^{1}$ Department of Pathology, University Hospitals Saint-Luc, Brussels, Belgium

${ }^{2}$ Department of Genetics, University Hospitals Saint-Luc, Brussels, Belgium

\section{Correspondence to}

Professor Louis Libbrecht, Department of Pathology, University Hospitals Saint-Luc, Brussels 1200, Belgium; louis. libbrecht@uclouvain.be

Received 14 February 2018 Revised 6 April 2018 Accepted 11 April 2018

Published Online First 27 April 2018
Check for updates

To cite: Libbrecht $L$, Baldin P, Dekairelle A-F, et al. J Clin Pathol 2018:71:743-744.

\begin{abstract}
Evaluation of molecular tumour heterogeneity relies on the tumorous nuclei percentage (TNP) assessment by a pathologist, which has been criticised for being inaccurate and suffering from interobserver variability. Based on the 'Big Bang theory' which states that KRAS mutation in colorectal cancer is mostly homogeneous, we investigated this issue by performing a critical analysis of the correlation of the KRAS mutant allele fraction with the TNP in 99 colorectal tumour samples with a positive KRAS mutation status as determined by next-generation sequencing. Our results yield indirect evidence that the KRAS zygosity status influences the correlation between these parameters and we show that a well-trained pathologist is indeed capable of accurately assessing TNP. Our findings indicate that tumour zygosity, a feature which has largely been neglected until now, should be taken into account in future studies on (colorectal) molecular tumour heterogeneity.
\end{abstract}

The Big Bang theory in colorectal cancer states that KRAS mutations are an early event in carcinogenesis and therefore mutations in this driver gene are frequently present throughout the tumour in a homogeneous fashion, while mutations in other genes such as BRAF and PIK3CA occur later and therefore these tend to be present heterogeneously. ${ }^{12}$ The studies that have led to this hypothesis are based on and take into account assessment of tumorous cell, or rather nuclei, percentage by a pathologist, since tumorous samples by definition do not contain tumorous nuclei and nuclei from non-tumorous cells such as myofibroblasts and lymphocytes. However, several studies have put forward that the assessment of tumorous nuclei percentage (TNP) by pathologists is not very accurate, showing considerable interobserver variability. $^{34}$

To further investigate this issue, we performed a critical analysis of the correlation between KRAS mutated allele frequency (MAF) and TNP assessed by a pathologist $(\mathrm{PB})$ in 99 colorectal cancer samples (primary tumours and metastatic sites in 89 and 10 patients, respectively) that had a positive KRAS mutation status as determined by next-generation sequencing (NGS) performed between January 2016 and June 2017. Scoring by the pathologist was done by eye balling using intervals of $10 \%$ and NGS was performed using the human tumour actionable mutation panel from Qiagen, which includes the $K R A S$ gene, on an Illumina MiSeq.

Mutations affecting exon 2 codons 12 and 13 accounted for $66 \%$ and $16 \%$, respectively, of all KRAS mutations found, in line with published positivity rates of different types of KRAS mutations in European patients with colorectal cancer.$^{5}$ As shown in Figure 1, there is a significant correlation between KRAS MAF and TNP, with a Pearson correlation coefficient of 0.3881 and a value of 0.000072 . By evaluating this graph and by looking at the raw data, with a MAF of more than $50 \%$ in several tumours suggesting that at least some tumours are not heterozygous, we speculated that differences in zygosity status might explain why this correlation, albeit present, was not very strong and why some tumours showed a TNP that was about the double of the MAF. For example, patient number 44 had a tumour with a MAF of $23 \%$ and a TNP of $50 \%$, while patient number 75 had also a MAF of $23 \%$ but a TNP of $20 \%$. Assuming that the former tumour is heterozygous for the KRAS mutation, the MAF has to be doubled to compensate for the heterozygosity which cannot be seen by the pathologist and the resulting new MAF is $46 \%$, which is close to identical to the TNP with a difference of only $4 \%$, while the difference before this adjustment was $27 \%$. The latter tumour is assumed to be homozygous for the KRAS mutation, hence the MAF is not changed and the difference between the MAF and the TNP therefore remains 3\%. We re-evaluated the MAF of all tumours and when the difference between the doubled MAF and the TNP was smaller than between the original MAF and the TNP, the doubled MAF was used as the new MAF and the tumour was considered as being heterozygous. By doing this, we assigned a heterozygous and homozygous status to $30 \%$ and $70 \%$ of tumours, respectively. The new $\mathrm{p}$ value was $<0.00001$ and the new correlation coefficient was 0.6912 , being almost the double of the original correlation coefficient. Compared with figures 1 and 2 indeed clearly depicts a much stronger correlation between the KRAS MAF and the TNP.

Our findings suggest that heterogeneity of KRAS mutation in colorectal cancer might actually even be less frequent than currently thought, because heterogeneity is deducted when a strong discrepancy between the MAF and the TNP is observed. ${ }^{26}$ However, difference in zygosity status 


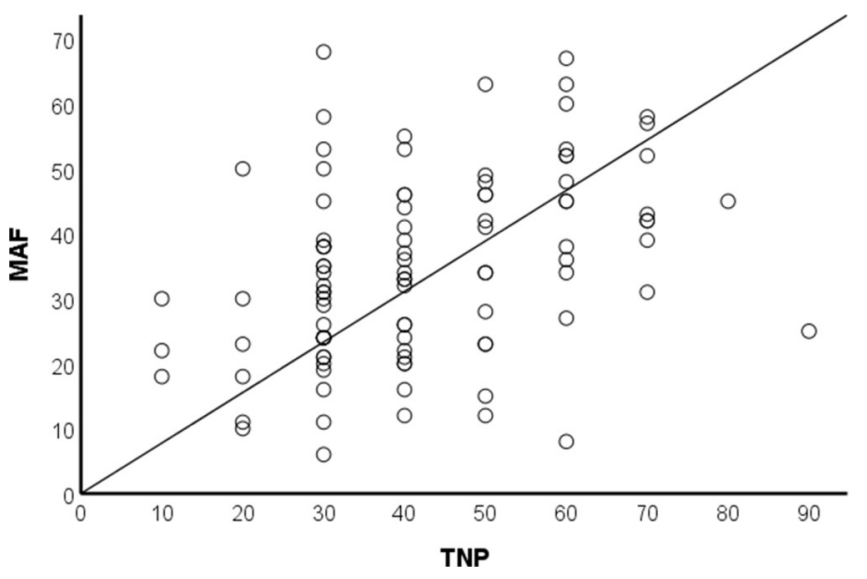

Figure $1 X-Y$ scatter plot showing the correlation between the tumorous nuclei percentage (TNP) and the KRAS mutant allele frequency (MAF).

between tumours might lead to a false impression of discrepancies. Therefore, our findings can be seen as a confirmation of the Big Bang theory. Moreover, our findings clearly show that a pathologist, even without the use of an zygosity status adjusted MAF, is indeed capable of performing a rather accurate assessment of TNP in a sample, in contrast with previous publications that were based on comparisons between pathologists, and not between a pathologist and the molecular data obtained from the samples. ${ }^{34}$ Thus, it is indeed useful to include TNP assessments in external quality control schemes.

A KRAS mutation zygosity analysis of 137 cell lines, including several of colorectal origin, showed that $37 \%$ was homozygous $^{7}$; in vivo data on the prevalence of homozygosity in colorectal cancer are currently not available. The assumption that colorectal tumours are per definition heterozygous and therefore the MAF for all tumours can be doubled when correlation with TNP is done since each nucleus contains two alleles ${ }^{2} 6$

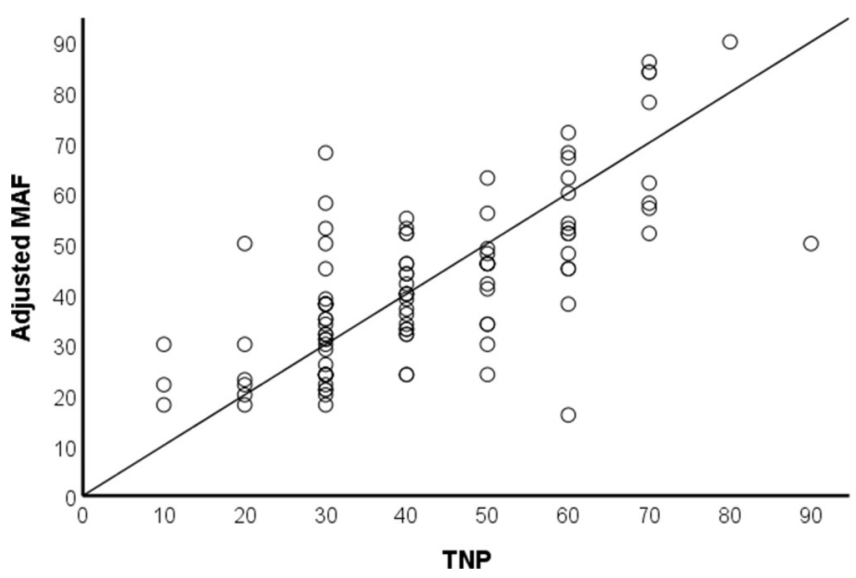

Figure $2 \mathrm{X}-\mathrm{Y}$ scatter plot showing the correlation between the tumorous nuclei percentage (TNP) and the adjusted KRAS mutant allele frequency (MAF) (see text for adjustment methods). should clearly be re-evaluated based on our data. Furthermore, KRAS mutation copy number gains due to amplification or polysomy or mutant allelic specific imbalance could also play a role, ${ }^{7}$ but if these phenomena would be present at a significant level in colorectal cancer, it would be impossible to find our reported correlation between KRAS MAF and TNP, either with or without the correction for presumed zygosity. Although we acknowledge that some assumptions are made and that our evidence is indirect, we feel that our findings are strong enough to propose that further research should address the issue of the zygosity status of KRAS and other mutations in colorectal cancer. Our findings could have consequences regarding the concept of heterogeneity in colorectal cancer and regarding the discussion on the accuracy of the pathologist's assessment of TNP in a sample.

\section{Take home messages}

- The data show that a well-trained pathologist is capable of accurately assessing tumoral nuclei percentage in the setting of colorectal cancer, supporting the use of such assesments in external quality assessment schemes.

- We find that the zygosity status of KRAS mutation probably influences the correlation between the KRAS mutant allele percentage and the tumoral nuclei assessment by the pathologist.

- Therefore, tumor zygosity, a feature which has largely been neglected until now, should be taken into account in future studies on (colorectal) molecular tumor heterogeneity.

\section{Handling editor Runjan Chetty.}

Contributors LL designed the study, performed statistical analyses and contributed to writing of the manuscript. A-FD performed the sequencing and revised the manuscript. PB performed the histological part of the study and revised the manuscript. AJ-M contributed to writing of the manuscript.

Competing interests None declared.

Ethics approval Ethics committee of the University Hospitals Saint-Luc.

Provenance and peer review Not commissioned; externally peer reviewed.

(c) Article author(s) (or their employer(s) unless otherwise stated in the text of the article) 2018. All rights reserved. No commercial use is permitted unless otherwise expressly granted.

\section{REFERENCES}

1 Sottoriva A, Kang H, Ma Z, et al. A Big Bang model of human colorectal tumor growth. Nat Genet 2015:47:209-16.

2 Normanno N, Rachiglio AM, Lambiase M, et al. Heterogeneity of KRAS, NRAS, BRAF and PIK3CA mutations in metastatic colorectal cancer and potential effects on therapy in the CAPRI GOIM trial. Ann Oncol 2015;26:1710-4.

3 Viray $\mathrm{H}$, Li K, Long TA, et al. A prospective, multi-institutional diagnostic trial to determine pathologist accuracy in estimation of percentage of malignant cells. Arch Pathol Lab Med 2013;137:1545-9.

4 Smits AJ, Kummer JA, de Bruin PC, et al. The estimation of tumor cell percentage for molecular testing by pathologists is not accurate. Mod Pathol 2014;27:168-74.

5 Boleij A, Tack V, Taylor A, et al. RAS testing practices and RAS mutation prevalence among patients with metastatic colorectal cancer: results from a Europe-wide survey of pathology centres. BMC Cancer 2016;16:825.

6 Haley L, Tseng LH, Zheng G, et al. Performance characteristics of next-generation sequencing in clinical mutation detection of colorectal cancers. Mod Pathol 2015;28:1390-9.

7 Soh J, Okumura N, Lockwood WW, et al. Oncogene mutations, copy number gains and mutant allele specific imbalance (MASI) frequently occur together in tumor cells. PLOS One 2009;4:e7464. 